\title{
Utilização de neuroimagem e eletroneurofisiologia por profissionais da área de saúde no atendimento de crianças com distúrbios da linguagem na cidade de Salvador-Bahia
}

\author{
Use of neuroimaging and electroneurophysiology by health professionals in the care of \\ children with language disorders in the city of Salvador - Bahia
}

\author{
Renata de Assis Fonseca Santos Brandão ${ }^{1 *}$, Ana Carla Filgueira de Souza e Souza ${ }^{1}$, Carlos Maurício \\ Cardeal Mendes², Eduardo Pondé de Sena ${ }^{3}$
}

\begin{abstract}
${ }^{1}$ Doutoranda do Programa de Pós-Graduação em Processos Interativos dos Órgãos e Sistemas, Instituto de Ciências da Saúde, Universidade Federal da Bahia. 2,3 Professor do Programa de Pós-Graduação em Processos Interativos dos Órgãos e Sistemas, Instituto de Ciências da Saúde, Universidade Federal da Bahia.
\end{abstract}

\section{Resumo}

Introdução: exames de neuroimagem e eletroneurofisiológicos possibilitam maior compreensão dos transtornos da linguagem. O objetivo deste estudo foi detectar como e quais desses exames são utilizados na avaliação clínica de crianças com alterações de linguagem por parte de médicos da cidade de Salvador (BA). Metodologia: os dados foram coletados por meio de questionário, com perguntas objetivas acerca dos exames. Resultados: a partir de bases de dados cedidas pelo Conselho Regional de Medicina da Bahia e pela Sociedade Baiana de Pediatria, contabilizaram-se 615 profissionais. Dentre eles, 345 estavam lotados em hospitais que dificultaram a realização da pesquisa. Ao final da coleta, foram contabilizados 31 questionários, preenchidos por pediatras (84\%), psiquiatras (3\%), neurologista (7\%) e neuropediatras (6\%). Das técnicas apresentadas, 91\% conhecem a tomografia computadorizada, $80 \%$ a ressonância magnética e $80 \%$ a eletroencefalografia. As técnicas de ressonância magnética funcional, potencial auditivo evocado, estimulação magnética transcraniana, tomografia por emissão de pósitrons e tomografia por emissão de fóton único são pouco conhecidas. Considerando os exames utilizados em crianças com distúrbios de linguagem, a tomografia computadorizada (7\%), a ressonância magnética (8\%), a eletroencefalografia (9\%) e a estimulação magnética transcraniana (1\%) foram os únicos citados, entretanto, $30 \%$ dos médicos relatam não prescrever os exames de neuroimagem e eletrofisiológicos. Conclusão: os resultados demonstram que esses exames são pouco utilizados. Dentre os utilizados e assinalados como disponíveis estão a tomografia computadorizada, o eletroencefalograma e a ressonância magnética.

Palavras-chave: Neuroimagem. Eletrofisiologia. Linguagem infantil. Transtornos da linguagem.

\section{Abstract}

Introduction: Neuroimaging and neurophysiological exams allow a better understanding of the evolution of language disorder. The aim of this study was to investigate which exams are used among doctors in Salvador-Bahia during their practices with children. Methodology: Data were collected using a survey with a closed-ended questionnaires, which inquired about the exams performed on patients with language disorder. Results: The database used was obtained from Conselho Regional de Medicina da Bahia and Sociedade Baiana de Pediatria and contained 615 doctors. After the exclusion criteria were applied, 31 surveys were completed and consisted of pediatricians (84\%), psychiatrist (3\%), neurologist (7\%), and neuropediatricians (6\%). Among the known techniques were: computer tomography (91\%), magnetic resonance (80\%), and electroencephalogram (EEG; 80\%) whereas other technics such as functional magnetic resonance (fMRI), P300, transcranial magnetic stimulation, positron emission tomography (PET), and single-photon emission computed tomography (SPECT) were less known. Amongst the doctors surveyed, only computer tomography (7\%), magnetic resonance (8\%), EEG (9\%), and transcranial magnetic stimulation (1\%) were prescribed for children with language impairment; whereas $30 \%$ of doctors reported never prescribing any complementary exams. Conclusion: Our results reveal that neuroimaging and electrophysiological exams were infrequently used among doctors surveyed; whereas computer tomography, EEG, and magnetic resonance were more frequently prescribed and accessible in Salvador-Bahia.

Keywords: Neuroimaging. Electrophysiology. Child language. Language disorders.

\section{INTRODUÇÃO}

A linguagem descrita e estudada por vários autores (BATES; DALE; THAL, 1995; BERGELSON; SWINGLEY, 2012;

Correspondente/Corresponding: *Renata de Assis Fonseca Santos Brandão. Programa de Pós-graduação Processos Interativos dos Órgãos e Sistemas/Instituto de Ciências da Saúde -UFBA. End: Avenida Reitor Miguel Calmon, s/n, 4ㅇandar, Sala 410, Vale do Canela, Salvador, Bahia, CEP: 40110-100. Tel:(71) 8872-3830
MILLS; COFFEY-CORINA; NEVILLE, 1993; TAYLOR, 2012) representa conceitos e intenções do locutor, seguindo convenções estabelecidas pela língua materna, podendo apresentar-se de diferentes formas. (DORE, 1974) A evolução do cérebro possibilita o desenvolvimento da capacidade linguística do ser humano e está intimamente relacionada a habilidades cognitivas diversas, envolvendo, 
portanto, várias áreas cerebrais. Determinadas lesões no sistema nervoso central (SNC) podem provocar o surgimento de distúrbios de linguagem. (POCA; MATARÓ, 2006)

Durante os primeiros anos de vida, o processo de maturação do encéfalo está sujeito, em grande parte, a mudanças secundárias e a estímulos ambientais. Apesar de, depois do nascimento, a quantidade de neurônios ser relativamente fixa, pode-se modificar a estrutura cerebral de acordo com os estímulos que procedem do meio em que a criança está imersa. Assim, durante esse tempo, o contexto que envolve a criança pode condicionar maior ou menor estrutura cerebral linguística. A infância é considerada uma época-chave para o desenvolvimento do SNC. (POCA; MATARÓ, 2006)

Transtornos da linguagem podem ser evidenciados como anormalidades no eletroencefalograma e em exames de neuroimagem e são identificados geralmente por volta dos três anos de idade. (PEREIRA; REIS; MAGALHÃES, 2003; CARTER, 2009)

Spironelli e Angrilli (2010) demonstraram que crianças podem apresentar frequência mais baixa de ondas, quando analisadas por meio do eletroencefalograma, ao serem comparadas a adultos durante a realização de tarefas fonológicas, semânticas e ortográficas. Além disso, verificaram que entre as crianças não há maturação necessária para se observar a lateralização dos processos linguísticos, como é observado em adultos.

Regan (1989) pontuou a importância do potencial evocado auditivo para avaliar discriminação, reconhecimento e categorização em indivíduos com incapacidades motoras e com alterações comportamentais. Hink, Hillyard e Benson (1978) e Alvarenga et al. (2013) observaram que, diante de estímulos linguísticos e tons puros, a presença da terceira onda lenta positiva (P300) foi maior em resposta aos estímulos linguísticos quando comparados aos tons puros. Além de ser observado em resposta a estímulos linguísticos, o P300 pode ocorrer durante estímulos visuais, solução de tarefas e respostas motoras ou decisões cognitivas. Estudo realizado por Alonso e Schochat (2009) identificou, pela avaliação do P300, melhora do processamento auditivo central em crianças, após treinamento auditivo. Leite, Wertzner e Matas (2010) também observaram melhora na amplitude do P300 em crianças com alterações fonológicas submetidas à terapia fonoaudiológica.

Não muito distante da utilização de dados eletrofisiológicos, como da eletroencefalografia e da análise de potenciais evocados, está a estimulação magnética transcraniana (EMT), que se baseia no princípio de indução eletromagnética de Faraday, cuja concepção considera que o campo magnético é gerado em virtude da passagem de corrente elétrica (BRUNONI; BOGGIO; FREGNI, 2012). Gough et al. (2012) utilizaram a EMT para verificação do potencial evocado motor em indivíduos durante nomeação de objetos. O resultado demonstrou aumento desse potencial durante a nomeação de itens conhecidos. Cacciari et al. (2011) utilizaram a mesma técnica para verificar o potencial motor evocado durante a leitura de frases literais, metafóricas, idiomáticas e figurativas, sendo que o potencial evocado foi maior durante a leitura de frases literais . Em estudo realizado por Liuzza, Candiditi e Aglioti (2011) em sujeitos saudáveis, notou-se que frases negativas tendem a inibir a via córtico-espinhal verificada pela estimulação magnética transcraniana. Busan et al. (2013) fizeram uso do EMT, pulso simples, para avaliar o potencial motor evocado de pacientes disfluentes e verificaram que o potencial evocado era menor quando comparado a grupo controle. Meister et al. (2003) observaram, pelo EMT no hemisfério dominante, que durante a leitura de uma e três palavras houve excitabilidade motora, padrão não detectado antes ou após leitura.

Além das técnicas eletrofisiológicas, dados de imagens registradas por meio de tomografia computadorizada (TC) e ressonância magnética (RM) também auxiliam no processo de diagnóstico e acompanhamento dos transtornos da linguagem. Caparulo et al. (1981) analisaram crianças com transtorno do espectro do autismo, alterações de linguagem, síndrome de Tourette e transtorno de déficit de atenção, utilizando TC, e encontraram $22 \%$ de exames anormais, porém, nenhuma relação foi encontrada entre gravidade dos sintomas e presença de anormalidades. Comparando o discurso de crianças após 3 anos de trauma cerebral com grupo controle, Wong et al. (2006) observaram, por meio de tomografia por emissão de fóton único (SPECT), hiperfusão nas regiões frontal esquerda, tálamo, globo pálido e putâmen à direita, lobos occipital e parietal bilateralmente, após realização de tarefa linguística. Quando combinados, os achados dos participantes com trauma em relação à perfusão e ao grau do discurso evidenciaram que a hipoperfusão do hemisfério direito está associada a baixo grau de performance discursiva, porém sugerem a necessidade de mais pesquisas com relação ao assunto, devido à utilização de pequena amostra no referido estudo. Gaillard et al. (2003) utilizaram ressonância magnética funcional (RMf) e verificaram ativação semelhante entre adultos e crianças, durante realização de tarefa semântica verbal. Ribaupierre et al. (2012) também utilizaram a RMf com sucesso para mapear as áreas corticais relacionadas à linguagem, em crianças antes de cirurgia para controle de epilepsia, evitando assim o teste funcional de Wada.

Do ponto de vista clínico, Scott (2012) pontuou a importância da utilização da tomografia por emissão de pósitrons (PET) e da RMf, durante o acompanhamento de pacientes com alterações de linguagem. Na prática fonoaudiológica, esses exames nem sempre são utilizados. Segundo a Lei federal 6.965/1981, não é permitido ao fonoaudiólogo solicitar nenhum tipo de exame complementar, podendo, porém, participar efetivamente de equipes de diagnóstico na área da comunicação humana. Cabe então à equipe médica realizar tais solicitações, nos casos de alterações da linguagem em crianças. Diante desse cenário, surgiu a necessidade de um levantamento com relação ao conhecimento e frequência com que são 
utilizados tais recursos diagnósticos pelos médicos pediatras, neuropediatras e psiquiatras infantis. Para tanto, foi elaborado um questionário, contendo perguntas acerca de exames eletroneurofisiológicos e de neuroimagem relacionados aos distúrbios da linguagem .

\section{MÉTODO}

Toda a pesquisa foi realizada de acordo com a norma 196/96 da Comissão Nacional de Ética em Pesquisa (CONEP), após a aprovação do Comitê de Ética em Pesquisa (CEP) da Maternidade Climério de Oliveira, sob o parecer 67133.

Todos os participantes incluídos na pesquisa assinaram o Termo de Consentimento Livre Esclarecido (TCLE), sendo que os profissionais que preencheram o formulário on line tiveram acesso à versão digitalizada do referido termo.

$\mathrm{O}$ acesso aos participantes ocorreu por meio de banco de dados cedidos pela Sociedade Baiana de Pediatria, pelo Conselho Regional de Fonoaudiologia (4ạ. Região Secção Bahia) e pelo Conselho Regional de Medicina. Também foram enviados e-mails aos membros da Associação de Neurologia, Psiquiatria e Áreas Afins (Sede Bahia), aos professores da Faculdade de Medicina da Bahia, da Universidade Federal da Bahia (constantes do site http://www.fameb.ufba.br), acessado em 26 de outubro de 2013, e dos membros da Associação de Neurologia (Capítulo Bahia).

Os dados cedidos pelas instituições citadas forneciam os nomes dos profissionais; apenas na lista fornecida pela Sociedade Baiana de Pediatria constava o telefone. Por meio do buscador Google, foram localizados os endereços. Aqueles que não foram encontrados na primeira busca - ou que não apresentavam telefone - foram excluídos. Os participantes incluídos foram contatados por telefone, com o objetivo de obter ou confirmar o endereço, assim como verificar os horários de disponibilidade para colaborar com a pesquisa. A partir desses dados, elaborou-se um roteiro para visitação a profissionais e instituições, por região de Salvador. Algumas áreas de Salvador, ao final da pesquisa, não foram visitadas, tendo portanto os profissionais atuantes nessas regiões sido excluídos da pesquisa.

Profissionais foram também acessados por e-mails enviados pela ABENEPI e pela Associação de Neurologia - Capítulo Bahia, localizados no site da Universidade Federal da Bahia.

A coleta foi realizada durante o período de agosto a dezembro de 2012. Vários profissionais foram localizados, via internet, em instituições hospitalares, motivo pelo qual elaborou-se ofício de apresentação, assinado pelo orientador e pelo coordenador do Programa de Pós-graduação de Processos Interativos dos Órgãos e Sistemas; foi aposto o documento de aprovação do Comitê de Ética e Pesquisa da Maternidade Climério de Oliveira. Endereço, telefone e/ou e-mail dos fonoaudiólogos registrados no Conselho Regional não foram encontrados na busca pela internet; obteve-se, assim, apenas uma listagem dos nomes dos profissionais nele registrados.

Os endereços dos consultórios particulares encontrados na internet foram visitados pelo menos duas vezes, após ligação telefônica para combinar horário e saber da disponibilidade e a confirmação de endereço. Quando a espera ultrapassava 30 minutos, considerou-se indisponibilidade para preenchimento do questionário; nestes casos, o profissional era excluído. Alguns questionários foram entregues para posterior preenchimento, com o consentimento do profissional ou de sua atendente.

Durante as ligações telefônicas, os profissionais que não mais trabalhavam com Pediatria também foram excluídos, assim como aqueles cujos telefones se encontravam desativados ou errados.

Para o estudo-piloto, 15 questionários foram aplicados aleatoriamente e algumas modificações foram realizadas, a partir das sugestões dadas pelos próprios profissionais respondentes.

O questionário, previamente elaborado para a pesquisa, incluiu dados sobre formação profissional, tempo e local de atuação. Inicialmente, continha 23 questões, sendo as últimas apenas para especialistas; devido à dificuldade de acesso e ao pequeno número de neuropediatras e psiquiatras infantis, essas questões foram descartadas. Foram utilizadas 13 questões objetivas relacionadas aos procedimentos diagnósticos de neuroimagem e eletroneurofisiologia cortical.

A análise estatística se desenvolveu em duas etapas. Na primeira, realizou-se a análise univariada dos dados dos profissionais, o que permitiu a obtenção da distribuição percentual e absoluta, mediante tabelas ou gráficos (coluna ou setor) das suas características sociodemográficas (sexo e faixa etária: 20-30, 31-40, 41-50, 51-60 e 61-70 anos), especialidades (Pediatria, Psiquiatria, Neurologia e Pediatria/Neurologia), conhecimento das técnicas de neuroimagem e eletroneurofisiologia e utilização de exames (TC, PET, SPECT, RM, RMf, P300, EMT e EEG), nos casos de distúrbios ou alterações da linguagem. Na segunda, as análises bivariadas permitiram verificar a associação do tipo de instituição, em relação ao tipo de especialidade do profissional de saúde, a qual foi recategorizada em Pediatria e Psiquiatria/Neurologia, e se a instituição na qual lecionava era de ensino superior pública ou de outra natureza. Para esse processo de análise estatística dos dados utilizou-se o programa STATA 12.1.

\section{RESULTADOS}

As bases de dados cedidas pelo Conselho Regional de Medicina da Bahia e pela Sociedade Baiana de Pediatria totalizaram 615 pediatras, neuropediatras e psiquiatras que seriam potenciais sujeitos; além destes, foram enviados 427 e-mails acessados no site da Faculdade de Medicina da Bahia, da Universidade Federal da Bahia.

Durante a fase de confirmação dos endereços, os telefones de 40 profissionais não foram localizados ou eles não atenderam as ligações. Dentre os profissionais 
contatados, 36 atuavam em outra especialidade, 59 apresentaram endereços errados, não foram encontrados ou foram visitados por 3 vezes sem êxito. Durante as visitas realizadas, 27 profissionais não estiveram disponíveis e 16 aceitaram receber o questionário para futuro preenchimento, porém a devolução não ocorreu em nenhum dos casos. Durante a procura de endereços, 345 profissionais foram encontrados em instituições hospitalares. Destes, 156 estavam lotados em instituições que negaram acesso e 189 tinham o endereço em instituições excluídas da coleta devido à dificuldade operacional imposta por elas próprias para a realização da pesquisa. Durante as visitas, 9 profissionais negaram o preenchimento e 60 não foram visitados. Os e-mails enviados em novembro de 2012 e junho de 2013 totalizaram 427, dentre os quais 7 registraram negação ao preenchimento, 14 retornaram, 8 responderam e 398 não responderam.

Dentre os profissionais que preencheram o questionário, $100 \%$ tinham diploma de especialização em Pediatria, Psiquiatria ou Neurologia.

O tempo médio de profissão foi de 21,12 anos entre os pediatras (dp 15,67 entre os pediatras e 8,04 para outras especialidades), 10 anos entre os psiquiatras, 18,5 anos entre os neurologistas $(d p=10,60)$ e 22 anos $(d p=$ $7,07)$ entre os neuropediatras.

Tabela 1 - Local de trabalho dos participantes - quantidade (porcentagem)

\begin{tabular}{lrcr}
\hline Tipo de instituição & Pediatra & $\begin{array}{c}\text { Psiquiatra/ } \\
\text { Neurologista }\end{array}$ & Total \\
\hline Privada & $5(19,23)$ & 0 & $5(16,13)$ \\
Pública & $3(11,54)$ & $1(20)$ & $4(12,90)$ \\
Privada e Pública & $12(46,15)$ & $4(80)$ & $16(51,61)$ \\
Não responderam & $6(23,08)$ & 0 & $6(19,35)$ \\
Total & $26(100)$ & $5(100)$ & $31(100)$ \\
\hline
\end{tabular}

Fonte: Dados da pesquisa.

Quadro 1 - Distribuição dos profissionais que lecionam em instituição de ensino superior

\begin{tabular}{|lcc|}
\hline \multicolumn{1}{|c|}{ Profissão } & \multicolumn{2}{c|}{$\begin{array}{c}\text { Leciona em Instituição } \\
\text { de Ensino Superior }\end{array}$} \\
Pediatria & Sim & Não \\
Psiquiatria & 7 & 19 \\
Neurologia & 0 & 1 \\
Neurologia/ Pediatria & 1 & 1 \\
Pearson chi2 =5,30 & 2 & 0 \\
Pr $=0,151$ & & \\
\hline
\end{tabular}

Fonte: Dados da pesquisa.

Com relação aos exames de neuroimagem e eletroneurofisiológicos, 32,26\% acreditam que a indicação desses exames na cidade de Salvador é fácil, enquanto
$64,52 \%$ discordam. Um participante não respondeu a questão (3,23\%). Entre os que discordam, 6,45\% trabaIham em instituições privadas, 6,45\% em instituições públicas, 32,25\% em ambas e 19,35\% não identificaram o tipo de instituição em que trabalhavam.

Gráfico 1 - Conhecimento acerca das técnicas

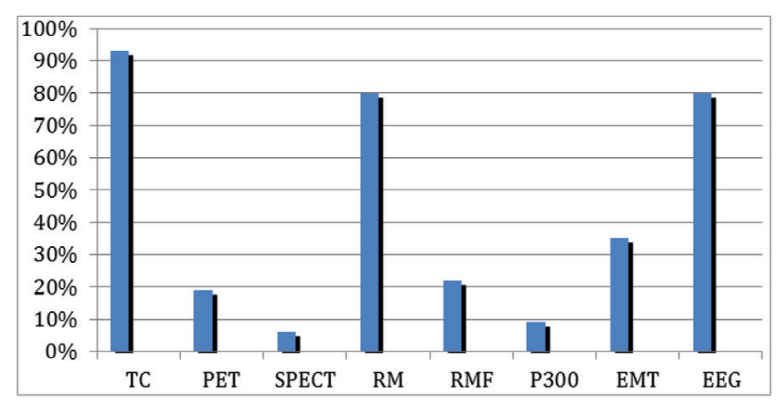

Fonte: Dados da pesquisa.

Gráfico 2 - Número de profissionais que utilizam exames nos casos de alterações de linguagem

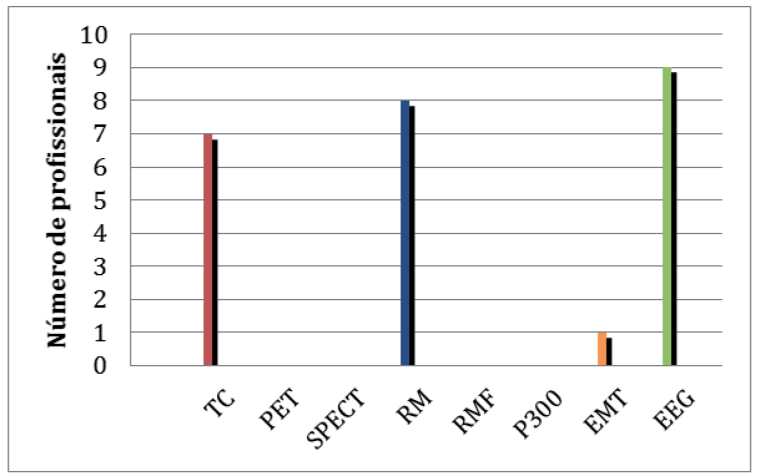

Fonte: Dados da pesquisa.

Com relação à indicação dos exames de neuroimagem e eletroneurofisiológicos em pacientes com alterações de linguagem, 7 pediatras solicitam tomografia computadorizada, no entanto, técnicas de PET e SPECT não foram assinaladas. A ressonância magnética é solicitada nos casos de alterações de linguagem por 3 pediatras e 5 psiquiatras ou neurologistas. A estimulação magnética transcraniana foi assinalada por 1 psiquiatra ou neurologista e o EEG foi escolhido como exame complementar por 6 pediatras e 3 psiquiatras ou neurologista. Ressonância magnética funcional e o P300 não foram mencionados por nenhum dos profissionais entrevistados.

Entre os profissionais que responderam ao questionário, a maior porcentagem para solicitação de exames encontrada foi "nunca", ou seja, os exames são solicitados com baixa frequência. 
Gráfico 3 - Frequência de solicitação de exames entre os profissionais

Frequencia de solicitação de exames entre os profissionais

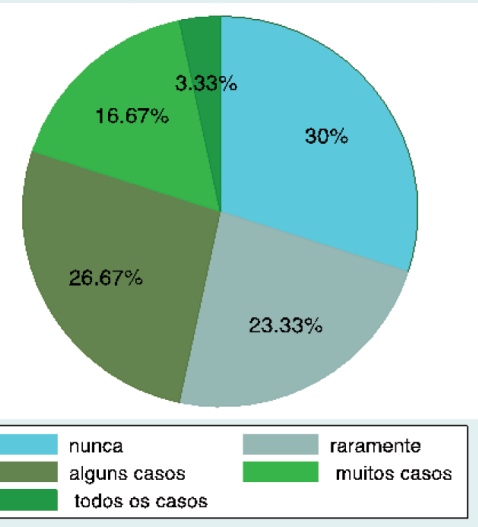

Fonte: Dados da pesquisa.

Mesmo não sendo solicitados com frequência exames para pacientes com alterações de linguagem, 38\% dos profissionais acredita que a TC é imprescindível para diagnóstico diferencial; $45 \%$ indicam a RM; $9 \%$, a RMF; 3\%, o P300; 6\% a EMT e 54\%, o EEG.

\section{DISCUSSÃO}

A utilização de questionários na área de saúde tem como objetivo a obtenção de maior reconhecimento acerca de determinado tema, sendo para saúde pública um instrumento que pode viabilizar prevenção e melhor acompanhamento de indivíduos. Dentre os tipos de questionários, estão os aplicados com questões abertas, mais discursivas, ou aqueles com questões fechadas. As questões fechadas possibilitam maior objetividade e facilidade na análise dos dados. Além da apresentação do questionário, a maneira como é aplicado pode viabilizar ou não a coleta. Esta pode ser realizada presencialmente, por telefone, por correspondência ou via internet. A opção pela realização presencial foi a busca de maior número de participantes, com a tentativa de reduzir ao máximo o viés de seleção. Leece et al. (2004) encontraram 58\% de respondentes via correspondência convencional, em seu estudo, enquanto via internet apenas $45 \%$ responderam. O mesmo ocorreu com Kim et al. (2000) que obtiveram apenas 9\% de respostas pela internet, enquanto que, por correspondência, foram 42\%. Leeuw (1992) destaca a realização presencial como superior naquela década, mesmo a correspondência convencional sendo, então, o meio mais utilizado.

Apesar de todo esforço para realização da coleta presencial para este estudo, vários médicos não se disponibilizaram a preencher o questionário ou o acesso a eles foi prejudicado por endereço errado ou recusa da instituição em colaborar com a pesquisa. Esta situação coincide com os dados apresentados por Nascimento Sobrinho et al. (2006), na cidade de Salvador, que resultaram em $42,3 \%$ de recusas e em $15 \%$ de perda por endereço errado, quando da aplicação de questionário para maior conhecimento do ambiente de trabalho dos médicos baianos. O mesmo aconteceu no estudo de Tucunduva et al. (2004), que obteve $41,7 \%$ de recusa na aplicação de questionário sobre prevenção e rastreamento de câncer.

Dentre os participantes, a grande maioria pertence ao sexo feminino (87\%), corroborando dados colhidos por Tucunduva et al. (2004), cujo estudo contou $57 \%$ de mulheres, diferentemente do encontrado por Nascimento Sobrinho et al. (2006), em questionários aplicados a médicos do programa Saúde da Família, os quais revelaram $54 \%$ de homens. O resultado desta pesquisa se explica pelo aumento de mulheres na profissão, segundo o portal do Conselho Federal de Medicina (CFM), ao informar que, desde 2006, houve um aumento de pessoas do sexo feminino. Além disso, a especialidade de Pediatria apresentou, em 2011, 70\% de mulheres atuantes (CONSELHO FEDERAL DE MEDICINA, 2011)

A maior frequência de médicos encontrada em instituições públicas e privadas difere dos dados fornecidos pelo Conselho Federal de Medicina (2011), o qual registra que $9,62 \%$ desses profissionais estão em instituições privadas e apenas $1,42 \%$, nas públicas.

De acordo com o mesmo Conselho (2011), 13,3\% dos médicos se encontram na especialidade de Pediatria, enquanto que em Neurologia e Psiquiatria a frequência cai, respectivamente, para 1,29\% e 3,44\%. Esses dados explicam a dificuldade de acessar especialistas nas áreas de Psiquiatria e Neurologia, principalmente aqueles que acompanham crianças.

Quando questionados sobre o conhecimento de exames de neuroimagem ou eletroneurofisiológicos, dos 31 participantes $80 \%$ responderam conhecer as técnicas de tomografia computadorizada, ressonância magnética e eletroencefalografia, enquanto poucos conheciam PET (19\%), SPECT (6\%), ressonância magnética funcional (22\%), P300 (9\%) e estimulação magnética transcraniana (35\%).

Esses achados vão de encontro aos achados científicos que relatam o uso de ressonância magnética funcional como elemento que pode contribuir para o diagnóstico e o acompanhamento de crianças (GAILLARD et al., 2003; ATTEVELDT et al., 2004; BONILHA et al., 2006; EYLER et al., 2012; RIBAUPIERRE et al., 2012); e de P300 (CHAPMAN et al., 1978; HINK; HILLYARD; BENSON, 1978; KUTAS; HILLYARD, 1980; REGAN, 1989; MUNHOZ et al., 2000; SKRANDIES; CHIU, 2003; ALONSO; SCHOCHAT, 2009; LEITE; WERTZNER: MATAS, 2010; ALVARENGA et al., 2013) Já o PET e SPECT são pouco conhecidos e dentre os exames complementares para distúrbios da linguagem são os menos utilizados, sugeridos apenas por Caparulo et al. (1981), Wong et al. (2006) Chugani et al. (2007). A estimulação magnética transcraniana é conhecida por $35 \%$ dos participantes, técnica esta que vem sendo estudada com maior especificidade desde a década de 1990. (FADIGA et al., 2002; MEISTER et al., 2003; ZADEH et al., 2004 ; ARAÚJO et al., 2011; PASCUAL-LEONE et al., 2011 ; BUSAN et al., 2013) 
Além da pequena frequência de conhecimento a respeito dos referidos exames dentre os participantes, observou-se baixa frequência na utilização desses exames diante de distúrbios da linguagem. O potencial evocado, P300, é utilizado apenas por um dos profissionais como exame complementar e na literatura é bastante descrito para acompanhamento de pacientes com alterações de linguagem (CHAPMAN et al., 1978; HINK; HILLYARD; BENSON, 1978; MOLFESE; HESS, 1978; KUTAS; HILLARD, 1980; REGAN, 1989; MUNHOZ et al., 2000; SKRANDIES; CHIU, 2003; ALONSO; SCHOCHAT, 2009; BURANELLI et al., 2009; LEITE; WERTZNER: MATAS; 2010; ALVARENGA et al., 2013)

Com base nos dados da literatura, os exames de neuroimagem e eletroneurofisiológicos podem contribuir no diagnóstico e acompanhamento de crianças com alterações de linguagem, porém, dos 31 participantes, $30 \%$ nunca se utilizam desses recursos no atendimento a pacientes com esse tipo de alteração. Mesmo não utilizando os exames, $38 \%$ dos participantes acreditam que a TC é imprescindível para diagnóstico diferencial; $45 \%$ optam pela RM; $9 \%$ indicam a RMf; $3 \%$, o P300; $6 \%$, a EMT; e $54 \%$, o EEG.

Durante a execução do projeto, houve algumas limitações, como dificuldade de acesso a instituições e falta de disponibilidade dos profissionais, fatores que resultaram em uma amostra pequena que pode apenas ser analisada descritivamente. Apesar dessas limitações, verificou-se que os exames complementares de neuroimagem e eletroneurofisiológicos são pouco utilizados no atendimento a pacientes que apresentam distúrbios de linguagem, por parte dos profissionais participantes da amostra, na cidade do Salvador. Além da não indicação, a ausência de conhecimento também prevaleceu, o que pode comprometer a qualidade da saúde pública, pois vários exames são utilizados para diagnóstico de outras patologias.

Dentre os exames utilizados, o EEG, a tomografia computadorizada e a ressonância magnética foram mais frequentes para o acompanhamento de crianças com alterações de linguagem.

Dentre os exames de neuroimagem e eletroneurofisiológicos, a tomografia computadorizada, a ressonância magnética e a eletroencefalografia foram assinaladas como disponíveis para utilização na cidade de Salvador.

\section{CONFLITO DE INTERESSE}

Não houve conflito de interesse durante a execução da pesquisa.

\section{REFERÊNCIAS}

1. ALONSO, R.; SCHOCHAT, E. A eficácia do treinamento auditivo formal em crianças com transtorno de processamento auditivo (central): avaliação comportamental e eletrofisiológica. Braz. J. Otorhinolaryngol., São Paulo, v. 75, n. 5, p. 726-732, 2009.

2. ALVARENGA, K. et al. The influence of speech stimuli contrast in cortical evoked potential. Braz. J. Otorhinolaryngol., São Paulo, v. 79, n. 3, p. 336-341, 2013.
3. ARAÚJO, H. A. et al. Estimulação magnética transcraniana e aplicabilidade clínica: perspectiva na conduta terapêutica neuropsiquiátrica. Revista de Medicina, São Paulo, v. 90, n. 1, p. 3-14, 2011.

4. ATTEVELDT, N. Van et al. Integration of letters and speech sounds in the human brain. Neuron., Cambridge, v. 43, n. 2, p. 271-282, 2004.

5. BATES, E.; DALE, P.; THAL, D. Individual differences and their implications for theories of language development. In: FLETCHER, P.; MACWHINNEY, B. (Eds.). The handbook of child language. Oxford: Basil Blackwell, 1995.

6. BERGELSON, E.; SWINGLEY, D. At 6-9 months, human infants know the meanings of many common nouns. Proc. Natl. Acad. Sci. U. S. A., Washington, v. 109, n.9, p.3253-3258, 2012.

7. BONILHA, L. et al. Speech apraxia without oral apraxia:can normal brain function explain the physiopathology? Neuroreport, New York, v. 17, n. 10, p. 1027-1031, 2006.

8. BRUNONI, A. R.; BOGGIO, P. S.; FREGNI, F. Estimulação elétrica no sistema nervoso central. In:______. Neuromodulação terapêutica: princípios e avanços da estimulação cerebral não invasiva em neurologia, reabilitação, psiquiatria e neuropsicologia. São Paulo: Sarvier, 2012. cap. 1, p. 3-20.

9. BURANELLI, G. et al. Verificação de respostas do mismatch negativity (MMN) em sujeitos idosos. Braz. J. Otorhinolaryngol.(Impr.)., São Paulo, v. 75 , n. 6 , p. $831-838,2009$.

10. BUSAN, P. et al. Motor excitability evaluation in developmental stuttering: a transcranial magnetic stimulation study. Córtex, Itália, v. 49, n. 3, p. 781-792, 2013. Disponível em: <http://www.ncbi.nlm.nih. gov/pubmed/22225881>. Acesso em: jul. 2013.

11. CACCIARI, C. et al. Literal, fictive and metaphorical motion sentences preserve the motion component of the verb: a TMS study. Brain lang., San Diego, v. 119, n. 3, p. 149-157, 2011.

12. CAPARULO, B. et al. Computed tomographic brain scanning in children with developmental neuropsychiatric disorders. J. am. acad. child Psychiatry, Baltimore, v. 20, n. 2, p. 338-357, 1981.

13. CARTER, R. The human brain book. London: Dorling Kindersley, 2009. p. $140-150$.

14. CERMARK, S. Developmental dyspraxia. In: ROY, E. A. Neuropsychological studies of apraxia and related disorders. Amsterdam: Elsevier, 1985. p. 225-248.

15. CHAPMAN, R. et al. Brain responses related to semantic meaning. Brain lang, New York, v. 5, n. 2, p. 195-205, 1978.

16. CHUGANI, H. et al. Autism with facial port wine stain: a new syndrome? Pediatr. neurol., New York, v. 37, n. 3, p.192-199, Sep.2007.

17. CONSELHO FEDERAL DE MEDICINA. Demografia médica no Brasil. São Paulo, 2011. v. 1.

18. DORE, J. A pragmatic description of early language development. J. Psycholinguist. res., New York, v. 3, n. 4, p. 343-350,1974.

19. EYLER, L.; PIERCE, K.; COURCHESNE, E. A failure of left temporal cortex to specialize for language is an early emerging and fundamental property to autism. Brain: J. neurology., London, v. 135, n. 3, p. 949960, 2012.

20. FADIGA, L. et al. Short communication: speech listening specifically modulates the excitability of tongue muscles: a TMS study. Eur. j. neurosci., Oxford, v. 15, p. 399-402, 2002. 
21. GAILLARD, W. et al. Developmental aspects of language processing: fMRI of verbal fluency in children and adults. Hum. brain mapp., New York, v. 18, p. 176-185, 2003

22. GOUGH, P. et al. Nouns referring to tools and natural objects differentially modulate the motor system. Neuropsychol., Oxford, v. 50, n. 1, p.19-25, 2012.

23. HINK, R.; HILLYARD, S.; BENSON, P. Event related potencials and selective attention to acoustic and phonetic cues. Biol. psychol., Amsterdam, v. 6, n. 1 , p. 1-16, 1978

24. KIM, H. et al. Use of new technology in endourology and laparoscopy by American urologists: internet and postal survey. Urology, Cleveland, v. 56, n. 5 , p. $760-765,2000$

25. KUTAS, M; HILLYARD, S. Reading senseless sentences: brain potentials reflect semantic incongruity. Science, New Series, Washington, v. 207, n. 4427, p. 203-205, 1980.

26. LEECE, P. et al. Internet versus mailed questionaire: a controlled comparision. J. med. internet res., Pittsburgh, v. 6, n. 4, p. 39, 2004.

27. LEEUW, E. D. de. Data quality in mail, telephone and face to face surveys. Amsterdam: TT - Publicaties, 1992

28. LEITE, R. A.; WERTZNER, H. F.; MATAS, C. G. Potenciais evocados auditivos de longa latência em crianças com transtorno fonológico. Pró-Fono, São Paulo, v. 22, n. 4, p. 561-566, 2010.

29. LIUZZA, M. T..; CANDIDI, M.; AGLIOTTI, S. M. Do not resonate with actions:sentences polarity modulates cortico-spinal excitability during action-related sentence reading. Plos One, San Francisco, v. 6, n. 2, p. e16855, 2011.

30. MEISTER, l. et al. Motor cortex hand área and speech: implications for the development of language. Neuropsychologia, Oxford, v. 41, p. 401-406, 2003.

31. MILLS, D.; COFFEY-CORINA, S.; NEVILLE ,H. Language acquisition and cerebral specialization in 20 month old infants. J. cogn neurosc., Massachusetts, v. 5, n. 3, p.317-334, 1993.

32. MOLFESE, D.; HESS, T. Hemispheric specialization for VOT perception in the preschool child. J. exp. child psychol., New York, v. 26, n. 1, p. 71-84, 1978.

33. MUNHOZ, M. L. et al. Potenciais evocados auditivos: aspectos históricos e técnicos. In: 2000. p. 165-167. . Audiologia clínica. São Paulo: Atheneu,

34. NASCIMENTO SOBRINHO, C. L. et al. Condições de trabalho e saúde mental dos médicos de Salvador, Bahia, Brasil. Cad. saúde pública, Rio de Janeiro, v. 22, n. 1, p. 131-140, 2006.
35. PASCUAL-LEONE, A. et al. CA - characterizing brain cortical plasticity and network dynamics across the age-span in health and disease with TMS-EEG and TMS-fMRI. Brain topogr., New York, v. 24, n. 3-4, p. 302315. 2011. Special edition: Brain imagin across the lifespan.

36. PEREIRA, J; REIS, A.; MAGALHÃES, Z. Neuroanatomia funcional: anatomia das áreas activáveis nos usuais paradigmas em ressonância magnética functional. Acta med. port., Lisboa, v. 16, p.107-116, 2003.

37. POCA, M. A.; MATARÓ, M. Desenvolvimento do cérebro e da linguagem - consequências do dano cerebral no cérebro imaturo. In: PLAJA, C. J.; RABASSA, O. B.; SERRAT, M. M. Neuropsicologia da linguagem: funcionamento normal e patológico. Santos: Reabilitação, 2006. p. 31-33.

38. REGAN, D. Human brain eletrophysiology: evoked potencials and evoked magnetic fields in science and medicine. Toronto: Elsevier, 1989.

39. RIBAUPIERRE, S. de et al. Presurgical language mapping in children with epilepsy: clinical usefulness of functional magnetic resonance imaging for the planning of cortical stimulation. Epilepsia, Malden, $v$. 53, n. 1, p. 67-78, 2012.

40. SCOTT, S. The neurobiology of speech perception and production-can functional imaging tell us anything we did not already know? J. commun. disord., London, v. 45, n. 6, p. 419-425, 2012

41. SKRANDIES, W.; CHIU, M. J. Dimensions of affective semantic meaning - behavioral and evoked potencial correlates in Chinese subjects. Neurosci. lett., Amsterdam, v. 341, p. 45-48, 2003.

42. SPIRONELLI, C.; ANGRILLI, A. Developmental aspects of language lateralization in delta, theta, alpha and beta EEG bands. Biol. psychol., Amsterdam, v. 85 , n. 2, p. 258-267, 2010

43. TAYLOR, C. L. Robustness, risk and responsivity in early language acquisition: a randomised trial of low intensity language promotion programme for slow to talk toddlers finds no effects on language or behavioural development. Evid. based med., London, v. 17, n. 4, p.109-110, 2012.

44. TUCUNDUVA, L. et al. Estudo da atitude e do conhecimento dos médicos não oncologistas em relação às medidas de prevenção e rastreamento do câncer. AMB rev. Assoc. Med. Bras., [online], São Paulo, v. 50, n. 3, 2004

45. WONG, S. et al. A SPECT study of language and brain reorganization three years after pediatric brain injury. Prog. brain res., Amsterdam, v. 157, p. 173-185, 2006

46. ZADEH, L.A. et al. Short Communication: Left hemisphere motor facilitation in response to manual action sounds. Eur. j. neurosci., Oxford, v. 19, p. $2609-2612,2004$

Submetido: 02/09/2015

Aceito em: 03/10/2015 